\title{
Stars at Low Metallicity in Dwarf Galaxies
}

\author{
Eline Tolstoy ${ }^{1}$, Giuseppina Battaglia ${ }^{2}$, and Andrew Cole ${ }^{3}$ \\ ${ }^{1}$ Kapteyn Institute, University of Groningen, the Netherlands \\ email: etolstoy@astro.rug.nl \\ ${ }^{2}$ European Southern Observatory, \\ Karl-Schwarzschild str. 2, Garching bei München, Germany \\ email: gbattagl@eso.org \\ ${ }^{3}$ School of Mathematics and Physics, University of Tasmania, \\ Hobart, Tasmania, Australia \\ email: andrew.cole@utas.edu.au
}

\begin{abstract}
Dwarf galaxies offer an opportunity to understand the properties of low metallicity star formation both today and at the earliest times at the epoch of the formation of the first stars. Here we concentrate on two galaxies in the Local Group: the dwarf irregular galaxy Leo A, which has been the recent target of deep HST/ACS imaging (Cole et al. 2007) and the Sculptor dwarf spheroidal, which has been the target of significant wide field spectroscopy with VLT/FLAMES (Battaglia 2007).
\end{abstract}

Keywords. galaxies: dwarf, galaxies: evolution, (galaxies:) Local Group

\section{Introduction}

Studies of individual low mass stars allow us to trace the properties of stars and thus the Interstellar Medium out of which they were formed back to the earliest times. Dwarf galaxies, which often have simple, small and predominantly old stellar populations, in principle, offer us the most straight forward route to the identification and study of these ancient stars. Dwarf galaxies are also presumed to be the most numerous type of object in the early Universe, to an even greater extent than they are today. If individual stars which formed before the Epoch of Reionisation, before the time when their light could reach us directly, can be identified as low mass stars today, they can provide one of the few probes of the "Dark Ages" of cosmic history and potentially offer unique information about which physical processes led to the re-ionisation of the Universe. Dwarf galaxies also offer us the opportunity to study low metallicity star formation much as it may have occurred in the early Universe.

The first challenge is to identify the oldest and least evolved stars in an ancient stellar population and the second is to put them into the context of the formation and evolution of the entire galaxy. This is easiest to attempt in the dwarf galaxies that are close enough for star formation histories (SFHs) to be accurately determined from Main Sequence Turnoff (MSTO) photometry and where follow-up spectroscopic abundances of the brightest ancient stars, the Red Giant Branch (RGB) stars, are possible. It is the combination of Colour-Magnitude Diagram (CMD) analysis and spectroscopic abundances of individual stars that allows us to most accurately determine the star formation and chemical evolution history of a galaxy from the earliest times. 


\section{Which dwarf galaxy?}

In the Local Group there is a large selection of dwarf galaxies which can in principle be used to study low metallicity star formation (e.g., Mateo 1998). The broad range of dwarf galaxy types is usually studied with quite different techniques, because their distances, sizes and surface brightnesses vary by a large amount. The properties and inter-relations of different types of dwarf galaxy are not always easy to understand when we must use different techniques to study them, and often observe different fractions of the stellar populations and over different fractions of the galaxy volume.

Is there a reason to study one dwarf galaxy rather than another? It of course depends on what you are looking for. Some dwarf galaxies still have on-going star formation at very low metallicity, presumably much as it was at the earliest times. But to find an actual star that was formed in the earliest times it is better to target galaxies containing predominantly ancient stars, so as not to have to look through more recent star formation episodes. On the other hand, some galaxies are just easier to study than others (due to distance, size, concentration, location in the sky, heliocentric velocity etc...). Here we give a brief overview of the different groupings of dwarf galaxy types in the Local Universe which can be well resolved into individual stars and of the techniques generally used to study them:

The ultra compact dwarf galaxies include actively star forming blue compact dwarf (BCD) galaxies (e.g., I Zw 18) and currently dormant dwarf ellipticals, dEs, (e.g., M 32) that are typically quite distant. The few dEs in the Local Group, are all to be found around M 31, 1 Mpc away, and there are no obvious BCDs (with the possible exception of IC 10, hidden behind significant foreground obscuration from the Milky Way). The compactness (and distance) typical of these systems means that resolved studies require the Hubble Space Telescope (HST).

Dwarf irregular galaxies (dIs) are numerous within the Local Group although they are typically at a distance $>400 \mathrm{kpc}$. These are HI-rich systems of varying sizes, masses and luminosities which are currently forming stars with a variety of rates, from extremely low (e.g., Pegasus) to almost zero (e.g., DDO 210) to very high (e.g., NGC 6822). Detailed studies down to the oldest MSTOs of these types of galaxies require HST-like sensitivity and image stability. Ca II triplet (CaT) surveys of their RGB populations are possible (although challenging).

Dwarf spheroidal galaxies (dSphs) are typically associated with large galaxies like our own, and so can be much closer than dIs, with the majority at distances $<130 \mathrm{kpc}$, although there are also several more distant examples. They look very much like the old extended stellar populations seen in dIs, and this suggests that the major difference is that they lack gas and recent star formation. They have typically not formed stars for at least several 100 Myr (e.g., Fornax), and in several cases much longer (e.g., Sculptor $\mathrm{dSph}$ formed the majority of its stars more than 10 Gyrs ago). The proximity of dSphs makes it easier to carry out spectroscopic studies of their resolved stellar populations, although this requires wide field instrumentation to efficiently gain an overview as they are typically $>1$ degree across on the sky. These galaxies have been the subject of much recent attention due to new wide-field multi-fibre spectroscopic facilities such as VLT/FLAMES and Magellan/MIKE.

There are also the newly discovered ultra faint systems, and it is not yet entirely clear that they are all galaxies. They are so faint and diffuse that they are only picked up by all sky surveys carried out with great care and attention to uniformity, such as SDSS (e.g., Belokurov et al. 2007, Martin et al. 2008). Often when their individual stars are spectroscopically followed up they are found to only contain a few RGB stars (if 


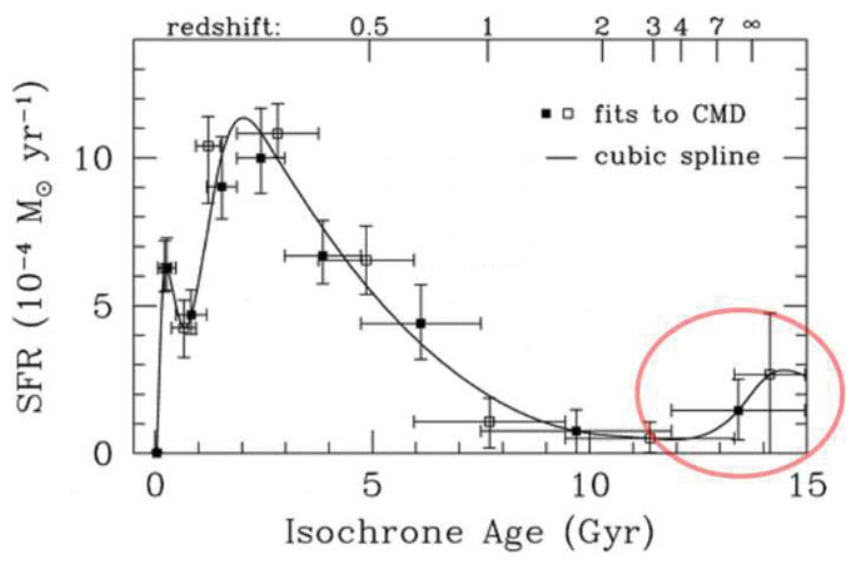

Figure 1. The derived SFH of Leo A, from Cole et al. (2007). The data points with $1 \sigma$ error bars show the best fits to the CMD. The heavy line is a spline fit to the results, and is the best estimate for Leo A's SFH. The circled area represents region of most interest to understand the early evolution of Leo A, and its relation to the Epoch of Reionisation. It will be best probed by studying the RR Lyr variable star population.

any) which makes separating them out from the Galactic foreground and determining their properties quite challenging (e.g., Siegel et al. 2008). In several cases it is almost impossible to obtain a statistically meaningful sample. There is evidence to suggest that their average metallicity is lower than any globular cluster, and also than other more luminous dwarf galaxies (Simon \& Geha 2007), and that the most metal-poor stars are to be found in these systems (A. Frebel, this volume).

To date, no one has made an equally careful study of the SFH (from CMD analysis) and a spectroscopic survey of the abundances of a large number of RGB stars in the same dwarf galaxy. HST is ideal for making deep and accurate CMDs of dIs (which are a good match to the field of view and sensitivity limits) but it sees only a very small fraction of a much larger dSph. It would require a dedicated survey effort even from wide field imagers on the ground to cover a sizeable fraction of their volume. Spectroscopic studies of individual stars on the other hand are more straightforward for the closer dSphs. Most dIs are too distant for FLAMES spectrosopic studies of their RGB, but there are smaller field multi-object spectrographs (e.g., FORS on VLT and DEIMOS on Keck) which, with a dedicated effort, could produce the medium resolution spectra for the reasonably sized samples of stars required.

Thus in the absence of one well studied galaxy we discuss two different systems, one with deep HST imaging (Leo A) by Cole et al. (2007) and one with wide-field spectroscopic VLT/FLAMES study (Sculptor dSph) by Battaglia (2007). Both studies give interesting (but different) insights into the evolution of dwarf galaxies.

\section{The Star-Formation History of Leo A}

The Leo A dI is still forming stars with a metallicity very similar to that of its oldest stars, and so it is a good probe of how star formation proceeds at very low metallicity, and also how the SFH varies over time in a relatively isolated system most likely depending predominantly upon internal processes.

Leo A is one of the least luminous, most metal-poor gas-rich galaxies in the Local Group (e.g., Mateo 1998). It is at a distance of $\sim 790 \mathrm{kpc}$ and it contains a small population of young, massive stars, with a current star formation rate of $1-2 \times 10^{-4} \mathrm{M}_{\odot} / \mathrm{yr}($ Hunter \& 
Elmegreen 2004). There are only a few small HII regions, and from spectroscopy of four of these, van Zee et al.(2006) determined an oxygen abundance of $12+\log (\mathrm{O} / \mathrm{H})=7.38 \pm 0.10$ (assuming solar $[\mathrm{O} / \mathrm{Fe}]$ this corresponds to $[\mathrm{Fe} / \mathrm{H}]=-1.5)$, which is one of the lowest ISM metal abundances known, and only slightly more metal rich than I Zw 18 (at $12+\log (\mathrm{O} / \mathrm{H})=7.20$, or $[\mathrm{Fe} / \mathrm{H}]=-1.7$ for solar $[\mathrm{O} / \mathrm{Fe}])$. Leo $\mathrm{A}$ is thus one of the least evolved gas-rich galaxies in the Local Group. There have been several past attempts to determine the SFH of this system (Tolstoy 1996; Tolstoy et al. 1998; Schulte-Ladbeck et al. 2002), but they all lacked deep MSTO photometry to enable an accurate determination of the SFH going back to the earliest times. Dolphin et al. (2002) provided the first definitive proof of truly ancient stars in Leo A with the detection of RR Lyrae variable stars.

As part of a major programme to study isolated Local Group dwarf galaxies, deep images of Leo A were obtained with the ACS on HST over 16 orbits by Cole et al. (2007). From the resulting CMDs, reaching down to apparent [absolute] magnitudes of $\left(\mathrm{M}_{475}, \mathrm{M}_{814}\right) \approx(29[+4.4], 27.9[+3.4])$, an accurate star formation rate as a function of time over the entire history of the galaxy was determined (see Fig. 1). From this we can see that $90 \%$ the star formation in Leo A happened during the last 8 Gyr. There was a peak in the star formation rate 1.5 - 3 Gyr ago, when stars were forming at a level 5-10 times the current rate. The CMD analysis of Leo A only requires a very slight metallicity evolution with time. The mean inferred metallicity in the past is consistent with measurements of the present-day gas-phase oxygen abundance.

There appears to have been only a small and uncertain amount of star formation in Leo A at the earliest times (highlighted as a circle in Fig. 1). The level determined from CMD analysis alone is clearly not very reliable as can be seen from the error bars. However, there is no doubt that it exists due to the presence of RR Lyrae variable stars (Dolphin et al. 2002). These previously discovered RR Lyrae were at the limit of detection with the WIYN telescope, but with the help of these new ACS data, additional RR Lyrae and more detailed information such as ages and metallicities can be obtained (Fiorentino, Saha et al. in prep.). Thus in Leo A it can be seen that some stars were formed around the epoch of re-ionisation, but the bulk were formed much later. Careful modelling and a more detailed look at current observations are required to understand the relation (if any) between the star formation rate at early times in Leo A and the events surrounding the re-ionisation of the Universe.

\section{Spectroscopy of Red Giant Branch Stars in Sculptor dSph}

From the point of view of picking out the most ancient populations in nearby galaxies, the diffuse Sculptor dSph, with no significant star formation over the last $10 \mathrm{Gyr}$, is a good target. Sculptor is a close companion of the Milky Way located at a distance of $\sim 80 \mathrm{kpc}$. Observations of the stellar population of Sculptor have revealed a sizeable population of RR Lyrae variable stars (e.g., Kaluzny et al. 1995), clearly indicating that its stellar population contains a globular cluster age component. Sculptor also shows tantalizing evidence in its extended horizontal branch for a chemical enrichment history consistent with extended early star formation episodes (e.g., Hurley-Keller et al. 1999; Majewski et al. 1999).

As part of the DART project (Tolstoy et al. 2006) a detailed photometric and spectroscopic survey of the Sculptor dSph was carried out (Tolstoy et al. 2004; Battaglia 2007; Battaglia et al. 2008a, Hill et al., in prep). The ESO/2.2m Wide Field Imager (ESO/WFI) was used to obtain photometry out to and beyond the nominal tidal radius to study the spatial properties of the resolved stellar population. VLT/FLAMES spectra 


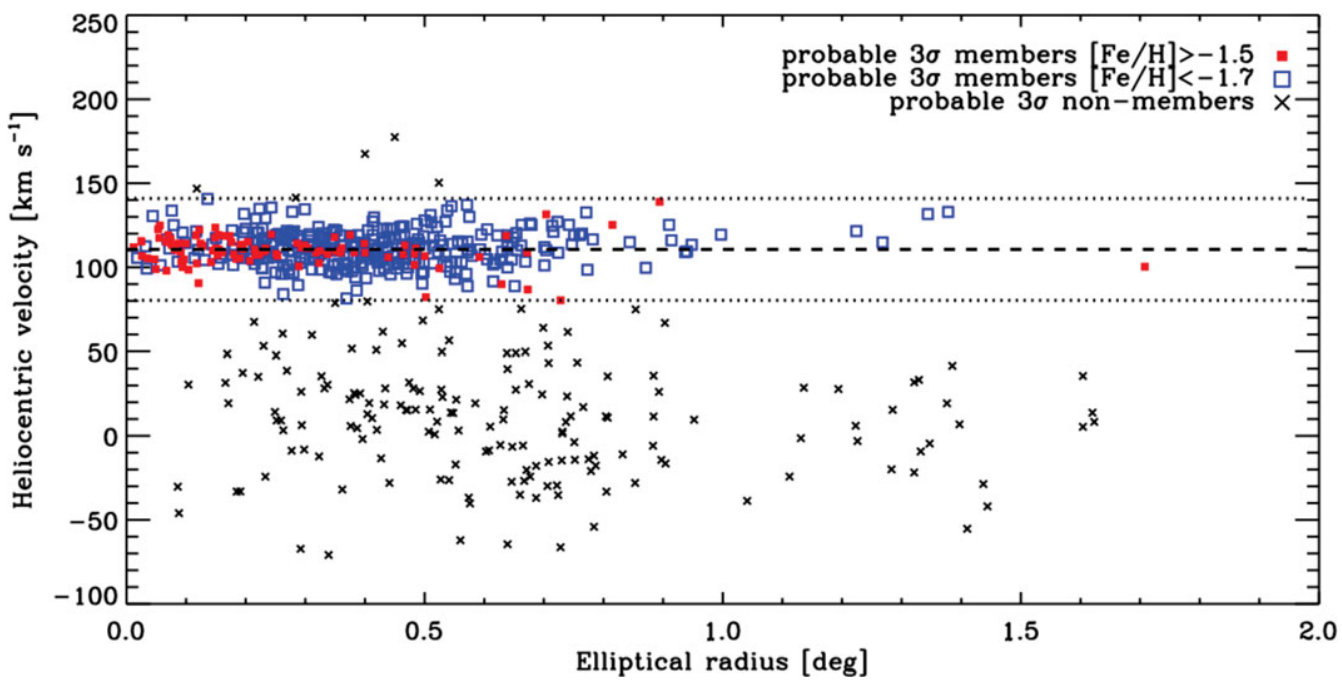

Figure 2. From the FLAMES LR sample of individual RGB stars in the Sculptor dSph (Battaglia 2007). The positions in the galaxy are given as Elliptical radii plotted against the heliocentric velocity of each star. The metal-rich stars $([\mathrm{Fe} / \mathrm{H}]>-1.5)$ are the solid (red) squares and the metal-poor stars $([\mathrm{Fe} / \mathrm{H}]<-1.7)$ are open (blue) squares, and the probable non-member stars are small (black) crosses, and the limits for membership are given by dotted lines about, $\mathrm{v}_{\text {hel }}=110.6 \mathrm{~km} / \mathrm{s}$ the heliocentric velocity (dashed line). The colder kinematics and centrally concentrated nature of the metal-rich stars are clearly visible compared to the metal-poor stars which have a larger velocity dispersion and are more uniformly distributed over the galaxy. Colours only available in the electronic version.

were obtained both at high resolution $(\mathrm{R} \sim 20000)$ for a detailed abundance analysis of $\sim 80$ RGB stars and at low resolution $(\mathrm{R} \sim 6500)$ in the CaT region for a sample of $\sim 470$ RGB stars.

The high resolution data consist of a single FLAMES pointing ( $25^{\prime}$ diameter) in the central $25^{\prime}$ of Sculptor which was observed for nearly 3 nights, and from the resulting spectra a detailed abundance analysis was carried out for a range of individual elements, including $\mathrm{Na}, \mathrm{Mg}, \mathrm{Ca}, \mathrm{Ti}, \mathrm{Fe}, \mathrm{Mn}, \mathrm{Ni}, \mathrm{Y}, \mathrm{Ba}$ and $\mathrm{Eu}$ (Hill et al., in prep.). This study allows a detailed picture of the chemical evolution of Sculptor from the earliest times.

The low resolution data comprise 15 FLAMES pointings distributed over the entire galaxy out to the nominal tidal radius. These provide accurate velocities and CaT metallicity estimates $([\mathrm{Fe} / \mathrm{H}])$, for a large fraction of the Sculptor RGB population (Battaglia 2007). A sample of 470 probable kinematic members of Sculptor was studied and stars of different metallicities were found to have different spatial distributions and kinematic properties, with the more metal-rich stars being more centrally concentrated and kinematically colder than the more metal-poor stars (see Fig 2). Signs of rotation were also found (Battaglia et al. 2008a), and the measured velocity gradient of $7.6_{-2.2}^{+3.0} \mathrm{~km} \mathrm{~s}^{-1}$ $\mathrm{deg}^{-1}$ makes the shape of Scl consistent with being flattened by rotation. Battaglia et al. 2008a also determined the mass of Sculptor within $1.8 \mathrm{kpc}$ using detailed mass modelling and find two separate components in Sculptor distinguished by metallicity, spatial extent and kinematics (Tolstoy et al. 2004). The new mass of Sculptor is largely independent of the exact distribution of dark matter in its central region, and with a $\mathrm{M} / \mathrm{L} \sim 160$, this gives a dynamical mass for the system, within $1.8 \mathrm{kpc}$, of $M_{\mathrm{dyn}}=3 \times 10^{8} M_{\odot}$, which is a factor $\sim 10$ higher than the previous value obtained from a much smaller and more centrally concentrated sample of stars (Queloz et al. 1995). 


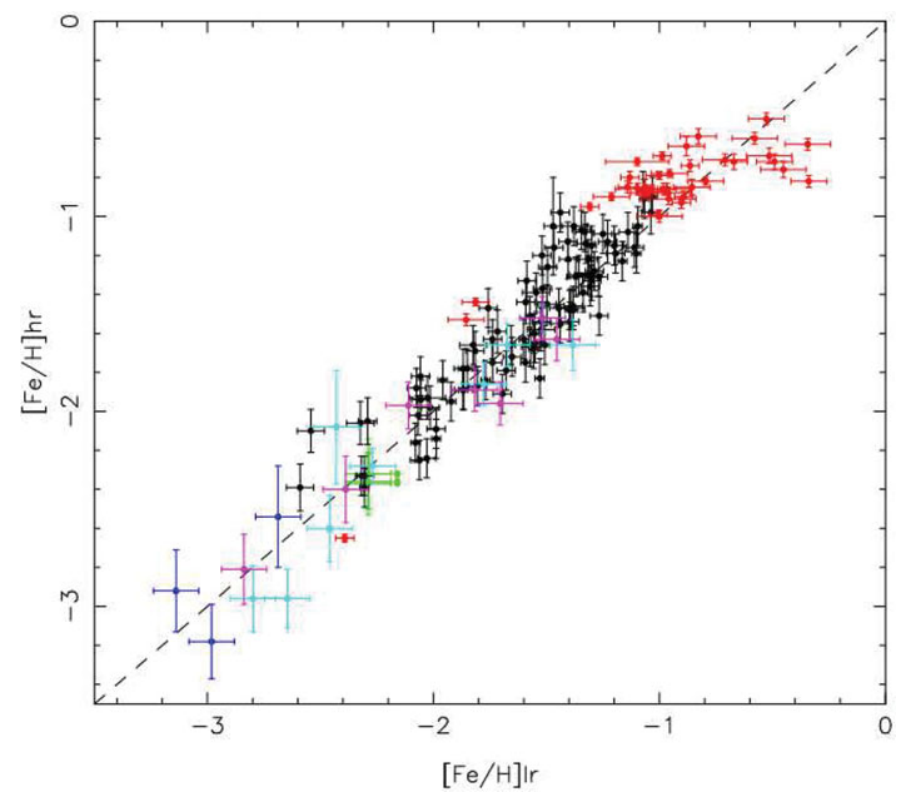

Figure 3. Low resolution ( $\mathrm{R} \sim 6000)$ CaT metallicity measurements of $[\mathrm{Fe} / \mathrm{H}]$ versus high resolution $(\mathrm{R} \geqslant 20000)$ direct measurements of $[\mathrm{Fe} / \mathrm{H}]$ from an average of $50+$ individual Fe lines. Included in this plot are HR and LR FLAMES/Giraffe data for Sculptor dSph (93 stars, black) and Fornax dSph (36 stars, red), from Battaglia et al. (2008b), and in addition follow-up spectroscopy with HET for 8 stars in Draco (cyan) and 7 stars in Ursa Minor (magenta) and 6 stars (green) in the globular cluster M 3 (Shetrone et al., in prep.) and 3 stars (dark blue) in Carina from MIKE/Magellan (Venn et al., in prep). Colours only available in on-line verison.

One useful advantage of studying a galaxy at both high and low resolution has been that we are able to compare in detail the values of $[\mathrm{Fe} / \mathrm{H}]$ determined from the CaT with those determined directly from large numbers $(50+)$ of individual Fe lines observed at higher resolution and also to take into account any potential effects due to differing $\alpha$-element abundances (Battaglia et al. 2008b). We find a very good match over the range of metallicities we have sampled (see Fig 3 ). This is quite a surprising result as it should be expected that the CaT method should stop working, or certainly the slope of the relation between the luminosity weighted equivalent width and $[\mathrm{Fe} / \mathrm{H}]$ ought to change. So far there has been no clear observational evidence for this, and theoretical studies are under way to try to understand when and how this method should stop working (e.g., Starkenburg, Hill et al., in prep.; see poster by E. Starkenburg, this volume).

In all our observations of the Sculptor dSph so far, we have not found any stars more metal poor than $[\mathrm{Fe} / \mathrm{H}] \sim-3$ (Battaglia 2007), and this is also true for the sample of 4 dSph galaxies looked at with VLT/FLAMES (Helmi et al. 2006). This lower limit is very similar to the lower limit observed in the Intergalactic Medium metallicities at redshifts, z $\sim 3$ (e.g., Cowie \& Songaila 1998). This could be interpreted as evidence that dSph galaxies formed out of an IGM at this redshift, however the presence and properties of the blue Horizontal Branch and RR Lyr variable stars, which are as ancient as those in globular clusters, would seem to contradict this. It may instead indicate that dSph galaxies played a significant role in the enrichment of the IGM before $\mathrm{z} \sim 3$. This is broadly consistent with the results of Ferrara \& Tolstoy (2000) who found that galaxies with (initial) gas masses of $\sim$ a few $10^{8} M_{\odot}$ are likely to be the major pollutants of the IGM because they will provide the maximum amount of mass (and metal) loss. This 
is a balance between limiting the losses so that the galaxy (and hence star formation) is not disrupted in a major way and not making the galaxy so big that little mass can be totally lost to the system. In this scenario galaxies that are so small that one star formation episode destroys the entire galaxy also do not get the opportunity to be significant contributors to the IGM. Sculptor certainly falls into the "blowout" category shown in Fig. 3 of Ferrara \& Tolstoy, which means that it loses gas and metals during star formation but not so much that it is immediately destroyed ("blowaway"). The presence of Sculptor in this regime may help to explain its early evolution.

\section{What have we learnt?}

The lack of very low metallicity stars in dSph suggests a threshold in the metallicity of the oldest stars in these galaxies. Interestingly there is some evidence that there are a couple stars with $[\mathrm{Fe} / \mathrm{H}]<-3$ in some of the ultra faint dwarf galaxies (A. Frebel, this volume). This combined with the fact that the low overall metallicities of these systems seem to fall into an overall mass-metallicity relation (Simon \& Geha 2007; H. Lee, this volume) suggests a global process which regulates the metallicity of a galaxy depending upon its mass. This mass-metallicity relation makes galactic scale winds the most likely culprit for the regulation of metallicity buildup in a small galaxy. Namely, due to these winds smaller galaxies are able to lose gas and metals more easily and so never form very many stars and those that they form are all of low metallicity.

There are, broadly speaking, three major types of faint dwarf galaxy in the vicinity of the Miky Way: 1) those interacting with our Galaxy, embedded in our halo (ultra faint dwarf galaxies); 2) those in the halo of our galaxy almost certainly affected dynamically by the presence our Galaxy (dSph); 3) more distant galaxies not obviously associated or ever having interacted with a large galaxy (dIs) and of course there are also intermediate "transition" types. All these systems may once have had very similar progenitors but the degree to which they have been influenced by interaction with our Galaxy varies strongly. It is of course very difficult to understand how different processes have affected these galaxies over a Hubble time as there is insufficient information to accurately trace back the history of these small systems in the context of the Milky Way and the Local Group partly because their 3D velocity is not known and partly because their original mass is also not known.

Every galaxy that we have looked at in sufficient detail to detect the Horizontal Branch - or with MSTO photometry (including Leo A and Sculptor) - clearly contains ancient stars. If this is not unambiguous from the CMD analysis it is then confirmed with detections of RR Lyr variable stars. The fraction of stars $>10$ Gyr old does vary considerably between different galaxies, but for all galaxies there is at least $\sim 10 \%$ of the stellar population that is ancient, and formed at a redshift $\mathrm{z}>3$. This still leaves some room for ambiguity about whether dwarf galaxies formed their first stars before or just after reionisation. However there is no evidence to suggest that the oldest stars in dwarf galaxies (including Sculptor) are any different (e.g., younger) than those in Galactic halo globular clusters, but it is very difficult to be precise about ages of stars $>10$ Gyr old. In the case of Sculptor an analysis of the well populated Horizontal Branch suggests that an age spread of $>2$ Gyr exists in this ancient population (Tolstoy et al. 2004) and this pushes the epoch at which the first stars could have formed in Sculptor back to at least 12-13 Gyr ago. Careful well calibrated MSTO analysis over the entire galaxy combined with spectroscopic abundances is required to address this issue.

Thus dwarf galaxies are clearly all ancient objects, well suited to test the properties of the early Universe. However it is difficult to make an accurate determination of the 
absolute ages of the oldest stars. It appears that we do not find extremely metal-poor stars in these systems and it is still not clear if this is due to pre-enrichment (they formed later than we presently believe) or if this is telling us something about the properties of the first stars to form in these systems (and hence in the Universe).

\section{References}

Battaglia, G., Helmi, A., Tolstoy, E., Irwin, M., Hill, V., \& Jablonka, P. 2008b, ApJL, 681, L13 Battaglia, G., et al. 2008a, MNRAS, 383, 183

Battaglia, G. 2007 Phd Thesis, University of Groningen, the Netherlands.

Belokurov, V., et al. 2007, ApJ, 654, 897

Cole, A. A., et al. 2007, ApJL, 659, L17

Cowie, L. L. \& Songaila, A. 1998, Nature, 394, 44

Dolphin, A. E., et al. 2002, AJ, 123, 3154

Ferrara, A. \& Tolstoy, E. 2000, MNRAS, 313, 291

Helmi, A., et al. 2006, ApJL, 651, L121

Hunter, D. A. \& Elmegreen, B. G. 2004, $A J, 128,2170$

Hurley-Keller, D., Mateo, M., \& Grebel, E. K. 1999, ApJL, 523, L25

Kaluzny, J., et al. 1995, ApJS, 112, 407

Majewski, S. R., Siegel, M. H., Patterson, R. J., \& Rood, R. T. 1999, ApJL, 520, L33

Martin, N.F., de Jong, J.T.A., \& Rix, H-W. 2008, ApJ, in press (arXiv:0805.2945)

Mateo, M. L. 1998, ARAA, 36, 435

Queloz, D., Dubath, P., \& Pasquini, L. 1995, A\& A, 300, 31

Schulte-Ladbeck, R. E., et al. 2002, AJ, 124, 896

Siegel, M. H., Shetrone, M. D., \& Irwin, M. 2008, AJ, 135, 2084

Simon, J. D. \& Geha, M. 2007, ApJ, 670, 313

Tolstoy, E., et al. 2006, The ESO Messenger, 123, 33

Tolstoy, E., et al. 1998, AJ, 116, 1244

Tolstoy, E. 1996, ApJ, 462, 684

Tolstoy, E., et al. 2004, ApJL, 617, L119

van Zee, L., Skillman, E. D., \& Haynes, M. P. 2006, ApJ, 637, 269

Walker, M. G., et al. 2007, ApJL, 667, L53 